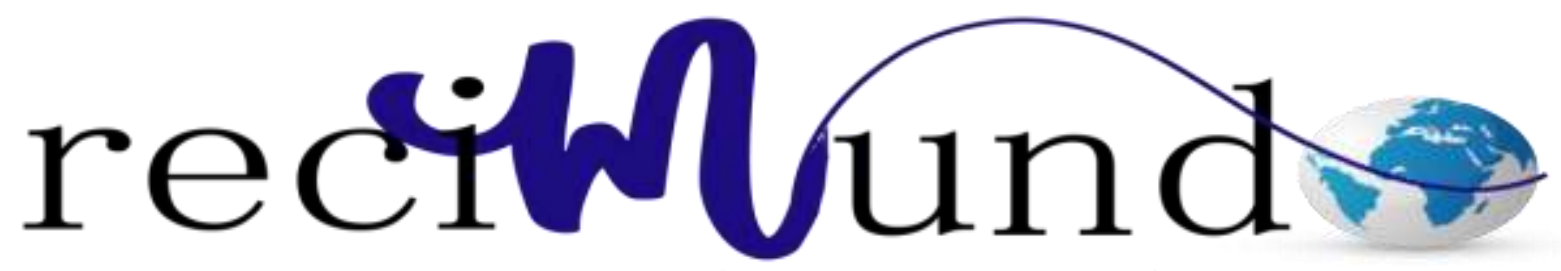

Revista Cientifica Mundo de la Investigación y el Conocimiento

Danixa Alexandra Rodríguez Chávez a; María Antonia Dominguez Zambrano ${ }^{\text {b; }}$ Mirian Mariana Basurto Macías ${ }^{\text {c; }}$ Yadira Geovana Yanes Tigselena ${ }^{\mathrm{d}}$

Distintos abordajes de clínicos quirúrgicos aplicando técnicas y procedimientos de acuerdo a criterios clínicos en oncología pediátrica

Different approaches of surgical clinicians applying techniques and procedures according to clinical criteria in pediatric oncology

Revista Científica Mundo de la Investigación y el Conocimiento. Vol. 3 núm. 4., diciembre, ISSN: 2588-073X, 2019, pp. 340-362

DOI: 10.26820/recimundo/3.(4).diciembre.2019.340-362

URL: http://recimundo.com/index.php/es/article/view/677

Código UNESCO: 3205 Medicina Interna

Tipo de Investigación: Artículo de Revisión

(C) RECIMUNDO; Editorial Saberes del Conocimiento, 2019

Recibido: 15/09/2019

Aceptado: 23/11/2019

Publicado: 30/12/2019

Correspondencia: karinaborjaa@gmail.com

a. Médico Cirujana; Investigadora Independiente; Guayaquil, Ecuador; dixy6891@ hotmail.com

b. Médico Cirujana; Investigadora Independiente; Guayaquil, Ecuador; dozamary@ hotmail.com

c. Médico; Investigadora Independiente; Guayaquil, Ecuador; mariana-b.m1990@ hotmail.com

d. Médico; Investigadora Independiente; Guayaquil, Ecuador; ygyanez.1212@ hotmail.com 


\section{Distintos abordajes de clínicos quirúrgicos aplicando técnicas y procedimientos de acuerdo a criterios clínicos en oncología pediátrica}

Vol. 3, núm. 4., (2019)

Danixa Alexandra Rodríguez Chávez; María Antonia Dominguez Zambrano; Mirian Mariana

Basurto Macías; Yadira Geovana Yanes Tigselena

\section{RESUMEN}

Los avances científico-técnicos de las últimas décadas han conducido a un aumento de la supervivencia en los niños enfermos de cáncer, los mismos, se sitúan por encima del 70\% a los cinco años del diagnóstico según sea el tipo de tumor, extensión y la edad del niño. En general, los tratamientos se caracterizan por ser largos, agresivos, con importantes efectos secundarios y modificadores de la vida familiar y del niño. En algunos casos pueden provocar secuelas irreversibles inmediatas. Todo ello, junto a la amenaza vital que representa un diagnóstico de este tipo, hace necesario el abordaje interdisciplinario que tenga por objeto conseguir un buen nivel de salud y una buena calidad de vida. Partiendo de una aproximación bio-psico-social de los procesos de salud y enfermedad; por tal razón, el psicólogo que trabaja en un servicio de onco-hematología pediátrica debería dar respuesta a las necesidades de cada niño. Dicho abordaje de manera general se divide en tres etapas: la primera, centrada en la adaptación a una enfermedad concreta, la segunda se refiere a las enfermedades crónicas en general y la tercera a la enfermedad oncológica, en la que aparecen aspectos propios de dicha enfermedad. A través de estas acciones, se pretende ayudar tanto a los padres como al niño recién diagnosticado a ir superando los sentimientos de indefensión, temor y confusión que caracterizan los momentos más difíciles generados por el mal oncológico diagnosticado, con el fin de que el grupo familiar, se sienta apoyado, ante las ansiedades de todas las personas implicadas en dicha situación previniéndose con ello, una mayor depresión, los efectos de la radioterapia en el sistema nervioso central, el conocimiento del niño sobre la enfermedad, la comunicación de malas noticias, la calidad de vida durante y después de la enfermedad, provocados por los procedimientos terapéuticos.

Palabras Claves: Criterios Clínicos; Oncología Pediátrica; Tratamiento Oncológico; Abordaje Clínico; Radioterapia. 


\section{Distintos abordajes de clínicos quirúrgicos aplicando técnicas y procedimientos de acuerdo a criterios clínicos en oncología pediátrica}

Vol. 3, núm. 4., (2019)

Danixa Alexandra Rodríguez Chávez; María Antonia Dominguez Zambrano; Mirian Mariana Basurto Macías; Yadira Geovana Yanes Tigselena

\section{ABSTRACT}

The scientific and technical advances of the last decades have led to an increase in survival in children suffering from cancer, they are above $70 \%$ five years after diagnosis according to the type of tumor, extent and age of the child In general, treatments are characterized by being long, aggressive, with important side effects and modifiers of family and child life. In some cases they can cause immediate irreversible sequelae. All this, together with the vital threat posed by a diagnosis of this type, makes the interdisciplinary approach necessary to achieve a good level of health and a good quality of life. Starting from a bio-psycho-social approach to health and disease processes; For this reason, the psychologist who works in a pediatric onco-hematology service should respond to the needs of each child. This approach is generally divided into three stages: the first, focused on adaptation to a specific disease, the second refers to chronic diseases in general and the third to oncological disease, in which aspects of the disease appear. Through these actions, it is intended to help both the parents and the newly diagnosed child to overcome the feelings of helplessness, fear and confusion that characterize the most difficult moments generated by the diagnosed cancer disease, so that the family group, he feels supported, in the face of the anxieties of all the people involved in this situation, preventing with it a greater depression, the effects of radiotherapy on the central nervous system, the child's knowledge about the disease, the communication of bad news, the quality of life during and after the disease, caused by therapeutic procedures.

Keywords: Clinical Criteria; Pediatric Oncology; Oncology Treatment; Clinical Approach; Radiotherapy. 


\section{Distintos abordajes de clínicos quirúrgicos aplicando técnicas y procedimientos de acuerdo a criterios clínicos en oncología pediátrica}

Vol. 3, núm. 4., (2019)

Danixa Alexandra Rodríguez Chávez; María Antonia Dominguez Zambrano; Mirian Mariana

Basurto Macías; Yadira Geovana Yanes Tigselena

\section{Introducción.}

El comportamiento de la célula cancerosa es distinto al de la célula normal, la misma tiene un crecimiento potencial indefinido, es capaz de invadir los tejidos circundantes y pueden diseminarse a distancia a otros puntos del organismo. Todas estas alteraciones son el resultado de las lesiones genéticas que producen alteraciones en la expresión de los genes o en su función bioquímica. En virtud de esto, el diagnóstico de cáncer puede ser considerado en general como un evento vital estresante que no sólo afecta al individuo que lo padece sino también a su entorno familiar y social más directo. (Aguirre, 2016).

El impacto que produce el cáncer no es el mismo en todos los individuos, si bien el proceso más común consiste en la valoración del evento como una amenaza, es muy probable que se genere una reacción emocional de ansiedad que puede llegar a ser muy intensa si el individuo percibe que sus recursos para afrontar la amenaza no son suficientes para controlar o manejar las consecuencias negativas que se presentan. La valoración del suceso como una pérdida importante, tenderá a producir una reacción de tristeza, que puede desencadenar en depresión. (Aguirre, 2016).

Entiéndase con esto que, la oncología pediátrica como una disciplina separada y unificada es muy joven. A través del siglo 20, el cuidado paliativo era considerado como la única opción, hasta el temprano éxito que se obtuvo en Estados Unidos con los medicamentos anticancerígenos impulsados por especialistas de los campos de la hematología, patología, radioterapia y cirugía, y algunos pediatras que investigaron sobre la naturaleza, prevención y tratamiento de tumores malignos en la infancia. Hasta hace algún tiempo, el cáncer infantil parecía 


\section{Distintos abordajes de clínicos quirúrgicos aplicando técnicas y procedimientos de acuerdo a criterios clínicos en oncología pediátrica}

Vol. 3, núm. 4., (2019)

Danixa Alexandra Rodríguez Chávez; María Antonia Dominguez Zambrano; Mirian Mariana Basurto Macías; Yadira Geovana Yanes Tigselena

ser una enfermedad relativamente rara y las causas de mortalidad infantil diferían bastante de las actuales. (Aguirre, 2016).

Anteriormente, las enfermedades infecciosas, las gastroenteritis severas con deshidratación, la patología neonatal, la prematuridad, las malformaciones congénitas, etc, ocupaban los primeros puestos como causas de muerte en la infancia. En la actualidad, el cáncer resulta ser la causa más influyente de muerte no accidental de niños con edades comprendidas entre los 2 y los 16 años. En el primer año de vida ocupa el tercer lugar de mortalidad, superada por las malformaciones congénitas. (Aguirre, 2016).

Por todo esto, es importante señalar que las enfermedades oncológicas representan importantes problemas de salud en todo el mundo, en Estados Unidos la incidencia anual de cáncer pediátrico asciende a 137 casos por un millón de habitantes, en Argentina se estiman 12 a 14 casos por cada 100000 niños menores de 15 años de edad2 y en el caso del área metropolitana de Bucaramanga, Colombia, 140 casos por un millón de habitantes en menores de 15 años. Hasta el $60 \%$ de los pacientes neutropénicos que tienen fiebre presentan una infección y el 16\% de las muertes a los cinco años del diagnóstico son por complicaciones de la quimioterapia, principalmente la infección. (Bradlyn, Beale, \& Kato, 2017).

El paciente oncológico se encuentra más expuesto a padecer infecciones debido a su estado de inmunosupresión, ya sea por los efectos producidos por su enfermedad de base o por el manejo instaurado para la misma. (Bradlyn, Beale, \& Kato, 2017). Durante el tratamiento antineoplásico los agentes citotóxicos son administrados junto con otras terapias inmunosupresoras, lo que produce efectos secundarios múltiples en el organismo, creando 


\section{Distintos abordajes de clínicos quirúrgicos aplicando técnicas y procedimientos de acuerdo a criterios clínicos en oncología pediátrica}

Vol. 3, núm. 4., (2019)

Danixa Alexandra Rodríguez Chávez; María Antonia Dominguez Zambrano; Mirian Mariana

Basurto Macías; Yadira Geovana Yanes Tigselena

alteraciones en el sistema inmune, tanto innato como adaptativo y favoreciendo la invasión por diferentes microorganismos. A nivel del sistema innato, se rompe la barrera cutánea con el uso de catéteres, se produce inflamación y lesión de las mucosas debido a la quimioterapia lo cual permite la invasión de agentes patógenos.

Adicionalmente, uno de los principales efectos es el compromiso a nivel de los neutrófilos, monocitos circulantes y macrófagos tisulares, en donde los agentes citotóxicos causan una alteración en la quimiotaxis, en la función bactericida, en la producción de agentes superóxidos y causan la disminución en el número de neutrófilos y monocitos; siendo así, la neutropenia una situación frecuente en estos pacientes. El resultado de estas alteraciones cuantitativas y funcionales es la incapacidad del sistema inmune de responder adecuadamente a la invasión por patógenos bacterianos y fúngicos, aumentando por tanto el riesgo de infección. (Bradlyn, Beale, \& Kato, 2017).

\section{Materiales y Métodos.}

\section{Fuentes documentales}

El análisis correspondiente a las características que predomina en el tema seleccionado lleva a incluir diferentes fuentes documentales encargadas de darle el respectivo apoyo y en ese sentido cumplir con la valoración de los hechos a fin de generar nuevos criterios que sirven de referencia a otros procesos investigativos. Para (Arias, 2010), las fuentes documentales incorporadas en la investigación documental o bibliográfica, "representa la suma de materiales sistemáticos que son revisados en forma rigurosa y profunda para llegar a un análisis del 


\section{Distintos abordajes de clínicos quirúrgicos aplicando técnicas y procedimientos de acuerdo a criterios clínicos en oncología pediátrica}

Vol. 3, núm. 4., (2019)

Danixa Alexandra Rodríguez Chávez; María Antonia Dominguez Zambrano; Mirian Mariana Basurto Macías; Yadira Geovana Yanes Tigselena

fenómeno". (p.41). Por lo tanto, se procedió a cumplir con la realización de una lectura previa determinada por encontrar aquellos aspectos estrechamente vinculados con el "CRITERIOS CLÍNICOS EN EL ABORDAJE DE LA ONCOLOGÍA PEDIÁTRICA" para luego explicar mediante un desarrollo las respectivas apreciaciones generales de importancia.

\section{Técnicas para la Recolección de la Información}

La conducción de la investigación para ser realizada en función a las particularidades que determinan a los estudios documentales tiene como fin el desarrollo de un conjunto de acciones encargadas de llevar a la selección de técnicas estrechamente vinculadas con las características del estudio. En tal sentido, (Arias Ob cit) refiere, que es "una técnica particular para aportar ayuda a los procedimientos de selección de las ideas primarias y secundarias”. (p. 71).

Por ello, se procedió a la utilización del subrayado, resúmenes, fichaje, como parte básica para la revisión y selección de los documentos que presentan el contenido teórico. Es decir, que mediante su aplicación de estas técnicas se pudo llegar a recoger informaciones en cuanto a la revisión bibliográfica de los diversos elementos encargados de orientar el proceso de investigación. Tal como lo expresa, (Arias Ob cit) "las técnicas documentales proporcionan las herramientas esenciales y determinantes para responder a los objetivos formulados y llegar a resultados efectivos" (p. 58). Es decir, para responder con eficiencia a las necesidades investigativas, se introdujeron como técnica de recolección el método inductivo, que hizo posible llevar a cabo una valoración de los hechos de forma particular para llegar a la explicación desde una visión general. 


\section{Distintos abordajes de clínicos quirúrgicos aplicando técnicas y procedimientos de acuerdo a criterios clínicos en oncología pediátrica}

Vol. 3, núm. 4., (2019)

Danixa Alexandra Rodríguez Chávez; María Antonia Dominguez Zambrano; Mirian Mariana

Basurto Macías; Yadira Geovana Yanes Tigselena

Asimismo, se emplearon las técnicas de análisis de información para la realización de la investigación que fue ejecutada bajo la dinámica de aplicar diversos elementos encargados de determinar el camino a recorrer por el estudio, según, (Arias, Ob cit) las técnicas de procesamiento de datos en los estudios documentales "son las encargadas de ofrecer al investigador la visión o pasos que debe cumplir durante su ejercicio, cada una de ellas debe estar en correspondencia con el nivel a emplear" (p. 123). Esto indica que, para llevar a cabo el procesamiento de los datos obtenidos, es necesario establecer las técnicas que serán seleccionadas, destacándose en este caso, de manera particular: fichas de resumen, textual, registros descriptivos entre otros, los mismos se deben ajustar al nivel que ha sido seleccionado.

\section{Resultados.}

\section{Oncología Pediátrica}

El cáncer infantil es poco frecuente. Representa del 3\% al 6\% de todas las enfermedades, sin embargo, es la segunda causa de muerte en niños entre 0 y 14 años de edad después de los accidentes". Los cánceres más frecuentes son las leucemias y los linfomas, seguidos de los tumores cerebrales que ocupan el segundo lugar. (Garreto, 2016). Otros tumores frecuentes son el neuroblastoma, el sarcoma de tejido blando y óseo, tumores renales, de células germinales y la retinoblastoma. Estadísticas del año 2013 en países latinoamericanos reportan 1.687 casos de cáncer en menores de 19 años, de los cuales 516 fallecieron, ocupando las leucemias y los linfomas el primer lugar, seguido de los tumores cerebrales. 


\section{Distintos abordajes de clínicos quirúrgicos aplicando técnicas y procedimientos de acuerdo a criterios clínicos en oncología pediátrica}

Vol. 3, núm. 4., (2019)

Danixa Alexandra Rodríguez Chávez; María Antonia Dominguez Zambrano; Mirian Mariana Basurto Macías; Yadira Geovana Yanes Tigselena

En las últimas décadas el pronóstico del cáncer infantil ha mejorado debido a los avances en tratamientos (gracias a estudios clínicos desarrollados a nivel internacional), a la mejoría de los métodos diagnósticos y a la conformación de equipos multidisciplinarios constituidos por médicos especialistas como radioterapeuta oncólogo, cirujano oncólogo, enfermeras preparadas en oncología pediátrica, además del hematólogo y el oncólogo pediatra. (Garreto, 2016). Hay diferencias notables entre el cáncer del niño y el del adulto. En la población infantil el cáncer no es prevenible, sólo el diagnóstico precoz es efectivo, mientras que para los adultos se dispone de herramientas de pesquisa como la mamografía, la exploración ginecológica y de los marcadores tumorales específicos. Hay tumores casi exclusivos en el niño como es el tumor de Wilms, la retinoblastoma y el meduloblastoma.

\section{Modalidades de Tratamiento}

La quimioterapia y la radioterapia en el niño traen efectos secundarios tardíos importantes como son los trastornos hormonales, déficit en el crecimiento, aparición de segundas neoplasias y deformaciones en el sistema músculo esquelético, ya que se trata de un individuo en crecimiento. En general, el tratamiento del cáncer en niños y adolescentes abarca tres grandes modalidades que son la cirugía, la radioterapia y la quimioterapia. (Marrown, 2017).

Por tal motivo, la atención centrada en el niño se basa en la evaluación del paciente, su educación, coordinación de la atención, cuidado directo y de apoyo. Entre esas demandas está el recibir el tratamiento en una unidad especializada con personal altamente calificado. En la evaluación del paciente, el personal del equipo de salud que trabaja con el niño y el adolescente con cáncer debe estar en capacidad de valorar el estado físico y emocional del niño, tener 


\section{Distintos abordajes de clínicos quirúrgicos aplicando técnicas y procedimientos de acuerdo a criterios clínicos en oncología pediátrica}

Vol. 3, núm. 4., (2019)

Danixa Alexandra Rodríguez Chávez; María Antonia Dominguez Zambrano; Mirian Mariana

Basurto Macías; Yadira Geovana Yanes Tigselena

conocimiento de la enfermedad, planificar el esquema terapéutico y elaborar una historia clínica detallada. (Marrown, 2017).

De allí que, una vez que se hace el diagnóstico del cáncer, la información debe transmitirse en un lenguaje claro, evitar contradicciones y nunca mentir. La comunicación es el elemento clave cuando se suministra el diagnóstico de cáncer. (Marrown, 2017). El lenguaje debe ser apropiado al nivel cultural y al estado de angustia del grupo familiar y del paciente; siempre se debe tener en cuenta que en momentos de estrés emocional los padres no siempre escuchan lo que el médico les dice, por lo tanto, la información debe repetirse varias veces para establecer el vínculo e iniciar el tratamiento. Así, el equipo que trabaja con el niño y adolescente con cáncer entra en un período educativo que se inicia antes de la terapia y continua durante y después de ésta.

Realizado el diagnóstico e informado a la familia del paciente el equipo especializado en la administración de quimioterapia dentro de la unidad debe implementar políticas y procedimientos para el uso seguro de los tratamientos, ya que generalmente se administran altas dosis de medicamentos antineoplásicos. El equipo debe vigilar y prevenir infecciones, para ello, quienes trabajan en el área de infusión, tanto enfermeras como médicos, deben hacer énfasis en el lavado de las manos y otros aspectos vitales en cuanto a la higiene. (Marrown, 2017). Una vez que se va a administrar el tratamiento, el equipo de salud debe vigilar ampliamente los efectos secundarios más frecuentes como son náuseas, vómitos y fatiga. Es importante comunicar a los padres lo concerniente a esos efectos antes de que aparezcan porque así se disipará la ansiedad del familiar y el equipo podrá prevenir los síntomas con las intervenciones adecuadas 


\section{Distintos abordajes de clínicos quirúrgicos aplicando técnicas y procedimientos de acuerdo a criterios clínicos en oncología pediátrica}

Vol. 3, núm. 4., (2019)

Danixa Alexandra Rodríguez Chávez; María Antonia Dominguez Zambrano; Mirian Mariana Basurto Macías; Yadira Geovana Yanes Tigselena

\section{Abordaje Clínico del Cáncer Infantil}

El cáncer es la proliferación continua e incontrolada de células anormales en el cuerpo.

Éstas en vez de responder a las señales que controlan el comportamiento celular normal, crecen y se dividen de manera incontrolada, invadiendo los tejidos y órganos sanos y, finalmente se diseminan por todo el cuerpo. (Marrown, 2017). De allí, que el cáncer se puede producir por la proliferación anormal de cualquiera de los diferentes tipos de células del cuerpo, por lo que existen más de cien tipos distintos de cáncer que difieren sustancialmente uno de otro en su comportamiento y respuesta al tratamiento.

Lo más importante a identificar en la patología del cáncer es distinguir entre los tumores benignos y malignos. Un tumor, es una proliferación anormal de células. Un tumor benigno, permanece confinado en su localización original, sin invadir el tejido sano adyacente ni propagarse a lugares distantes del cuerpo. Un tumor maligno, por su parte, es capaz de invadir el tejido normal adyacente y de propagarse por el cuerpo mediante los sistemas circulatorio o linfático (metástasis). Sólo a los tumores malignos se les denomina propiamente como cáncer, y es la capacidad que tienen para invadir y dar lugar a metástasis lo que los hace tan peligrosos. (Bestiet, 2017).

Tanto los tumores malignos como los benignos se clasifican de acuerdo al tipo de célula del que proceden. La mayoría de los cánceres se incluyen en uno de los tres tipos principales: carcinomas, sarcomas y leucemias o linfomas. Los carcinomas que incluyen aproximadamente el 90\% de los cánceres humanos, son alteraciones de las células epiteliales. (Bestiet, 2017). Los sarcomas, son tumores sólidos de tejidos conectivos como el músculo, hueso, cartílago y tejido fibroso. Las leucemias y los linfomas surgen a partir de las células hematopoyéticas y de las células 


\section{Distintos abordajes de clínicos quirúrgicos aplicando técnicas y procedimientos de acuerdo a criterios clínicos en oncología pediátrica}

Vol. 3, núm. 4., (2019)

Danixa Alexandra Rodríguez Chávez; María Antonia Dominguez Zambrano; Mirian Mariana

Basurto Macías; Yadira Geovana Yanes Tigselena

del sistema inmune respectivamente. Estos tumores se clasifican a su vez atendiendo al tejido de origen y al tipo de célula involucrada. Los cánceres más comunes que constituyen más de la mitad de los casos de cáncer son los de mama, próstata, pulmón y colon/recto.

La iniciación del tumor se considera que se debe a una alteración genética que provoca la proliferación anormal de una única célula. La proliferación celular da lugar a una población clonal de células tumorales. (Bestiet, 2017). La progresión del tumor se produce a medida que se generan mutaciones adicionales en las células de la población del tumor. Algunas de estas mutaciones confieren una ventaja selectiva a la célula como, por ejemplo, un crecimiento más rápido y los descendientes de las células que portan dicha mutación dominarán en la población tumoral. A este proceso se le denomina selección clonal, puesto que un nuevo clon de células tumorales evoluciona en función de su ritmo de crecimiento más rápido o de otras propiedades, que le confieren una ventaja selectiva.

Desde esta perspectiva se considera importante señalar que, la detección del cáncer en niños es a veces muy difícil de detectar. Los padres deben esforzarse por ir con sus hijos a exámenes médicos regulares y deben mantenerse alerta ante cualquier signo o síntoma inusual que sea persistente. (Bestiet, 2017). Entre los signos o síntomas persistentes que se deben tener en cuenta se encuentran los siguientes:

- Masa o tumefacción extraña.

- Palidez inexplicable.

- Pérdida de energía. 


\section{Distintos abordajes de clínicos quirúrgicos aplicando técnicas y procedimientos de acuerdo a criterios clínicos en oncología pediátrica}

Vol. 3, núm. 4., (2019)

Danixa Alexandra Rodríguez Chávez; María Antonia Dominguez Zambrano; Mirian Mariana Basurto Macías; Yadira Geovana Yanes Tigselena

- Tendencia de repente a la formación de hematomas.

- Dolor o cojera persistente, localizada.

- Fiebre o enfermedad prologada, inexplicada.

- Dolores de cabeza frecuentes, en algunos casos con vómitos.

- Cambios repentinos en los ojos o la visión.

- Pérdida excesiva y rápida de peso.

Cuando el diagnóstico de cáncer en el niño ya se ha comprobado, esto resulta ser una de las experiencias más dolorosas y estresantes por las que se tienen que enfrentar los niños y sus familias; de allí, que su abordaje se establece a través de los siguientes pasos:

Sintomatología y Tratamiento: La sintomatología ha sido uno de los aspectos más estudiados por teóricos, pues en gran parte éstos se relacionan con la calidad de vida del paciente oncológico y de sus familiares luego de haberse emitido un diagnóstico. El control de los síntomas es una parte esencial de la atención integral al niño que se encuentra gravemente enfermo o de los cuidados paliativos en pediatría. El objetivo principal de éste es prevenir y aliviar el sufrimiento en todas las dimensiones: física, emocional y espiritual.

Los síntomas están compuestos por múltiples estados que tienen diversos significados. Por esto, al experimentarse los síntomas como estados globales, se hace difícil aislar unos de otros para poder evaluar el grado de intensidad de cada uno. Esto contribuye a que los niños presenten dificultades o muestren desinterés a la hora de hablar sobre sus síntomas con otras personas, sobre todo cuando se conceptualizan como efectos secundarios o estados fisiológicos o psicológicos 


\section{Distintos abordajes de clínicos quirúrgicos aplicando técnicas y procedimientos de acuerdo a criterios clínicos en oncología pediátrica}

Vol. 3, núm. 4., (2019)

Danixa Alexandra Rodríguez Chávez; María Antonia Dominguez Zambrano; Mirian Mariana

Basurto Macías; Yadira Geovana Yanes Tigselena

singulares. Además, los niños pueden verse afectados por más de una experiencia de síntomas a la vez. (Bestiet, 2017).

Entre los efectos colaterales presentados por los tratamientos de cáncer se encuentran los siguientes: fatiga, náuseas, vómito, dolor y ardor en el sitio de la veno punción, reacciones alérgicas, pérdida del cabello, úlceras bucales, constipación, bajo conteo de células, ictericia, cistitis hemorrágica y cambio en el sistema nervioso, entre otros. Esto se debe a que los medicamentos del cáncer no sólo afectan a las células cancerosas sino también a otras células normales que se dividen rápidamente tales como las del tracto gastrointestinal, la médula ósea, los folículos pilosos y las células reproductoras. (Bestiet, 2017).

Prevalencia del Dolor en Oncología Pediátrica: De acuerdo con la definición de la Asociación Internacional para el Estudio del Dolor IASP (2016), el dolor es una experiencia sensorial y emocional desagradable asociada a una lesión presente o potencial o descrita en términos de la misma. Junto a la anorexia y la fatiga, el dolor es uno de los síntomas más comunes asociados al cáncer. El dolor no sólo depende del daño físico, ya que el mismo tipo de lesión tisular puede causar dolor de diferente naturaleza o intensidad en diferentes niños o en el mismo niño en diferentes momentos. (Bestiet, 2017).

Es por esto, que resulta indiscutible que la subjetividad inherente del dolor es un impedimento fundamental para poder conocer en detalle sus mecanismos y su control. Aunque no se dispone de estadísticas mundiales referentes a los diferentes tipos de dolor en niños oncológicos, los centros de tratamiento señalan que, en países desarrollados, los niños con cáncer experimentan dolor debido a la enfermedad y/o tratamiento. Entre las causas más importantes se señalan los 


\section{Distintos abordajes de clínicos quirúrgicos aplicando técnicas y procedimientos de acuerdo a criterios clínicos en oncología pediátrica}

Vol. 3, núm. 4., (2019)

Danixa Alexandra Rodríguez Chávez; María Antonia Dominguez Zambrano; Mirian Mariana Basurto Macías; Yadira Geovana Yanes Tigselena

procedimientos médicos diagnósticos y terapéuticos, hasta el punto de que muchos niños han considerado estas intervenciones mucho peores que la misma enfermedad. (Bestiet, 2017).

El dolor es uno de los síntomas más comunes que representa el mayor problema experimentado en los tratamientos realizados en las diferentes fases del cáncer y es una de las mayores preocupaciones de los padres. En el diagnóstico o durante el proceso del tratamiento, aproximadamente el 50\% de los niños con cáncer experimentan algún dolor. El dolor por cáncer puede ser agudo o crónico, puede tener características del dolor nociceptivo, neuropático, visceral o mixto u manifestarse como un síndrome específico o como un síndrome doloroso regional complejo. (Bestiet, 2017).

El dolor no aliviado, puede interferir en el sueño, produce fatiga y sentimientos de impotencia, lo cual puede llevar al aumento de morbilidad y mortalidad en niños. En efecto, el dolor debe ser considerado como un síntoma importante que debe ser evaluado y diagnosticado. El personal de salud debe considerar los diferentes componentes y causas del dolor para así poder realizar un tratamiento individualizado para cada paciente y familia, que a su vez permita ser monitoreado y ajustado con la finalidad de obtener la mejor eficacia analgésica con el menor nivel de efectos adversos. Por tal motivo, las drogas son consideradas clave en el manejo del dolor. Los niños con dolor severo requieren potentes analgésicos que les alivien dicha molestia y una apropiada administración de opioides que no los lleve a crear adicción por estos. (Bestiet, 2017).

En algunos casos, los mecanismos del dolor pueden influenciar el efecto analgésico de los opioides. La necesidad de utilizar altas dosis de opióides en casi todos los pacientes está asociada con la propagación del tumor a algunas áreas específicas del sistema cerebral como el 


\section{Distintos abordajes de clínicos quirúrgicos aplicando técnicas y procedimientos de acuerdo a criterios clínicos en oncología pediátrica}

Vol. 3, núm. 4., (2019)

Danixa Alexandra Rodríguez Chávez; María Antonia Dominguez Zambrano; Mirian Mariana

Basurto Macías; Yadira Geovana Yanes Tigselena

peri-acueducto gris, las raíces de los nervios espinales, los plexos nerviosos o la compresión de la columna vertebral. Los efectos secundarios de los opióides se deben anticipar y tratar antes que se presenten. Algunos de los efectos que pueden presentarse son náuseas, vómitos, constipación, etc., y pueden agravarse por la naturaleza y progresión del cáncer. (Bestiet, 2017). Entre los aspectos Temporales del Dolor se encuentra:

- Dolor Constante: este dolor está asociado a la administración de analgésicos, tiene mayor relación con el tiempo transcurrido entre cada toma que entre los síntomas que se presentan. Su mejor manejo es con la toma de analgésicos de alta duración por lo que se debe adecuar el tiempo de vida medio de cada medicamento pues si se produce la toma del medicamento sin el arribo del dolor se previne su recurrencia.

- Dolor Incidental: está relacionado con actividades específicas tales como comer, defecar, socializar o caminar, entre otras. Se presenta cuando la concentración del analgésico en sangre disminuye causando un mínimo efecto analgésico. Este se trata incrementando la dosis del analgésico o reduciendolos intervalos entre toma.

- Dolor Intermitente: este dolor es muy impredecible por lo que la mejor forma de manejarlo es mediante la administración de un potente analgésico mientras ocurre el dolor.

En dolor por cáncer la meta es la calidad de vida del paciente e implica el alivio del dolor y de los otros síntomas aun sin que ellos representen una mejoría de su funcionalidad que en muchos casos va a estar determinada por la progresión de la enfermedad de base. De ahí, que el pilar del manejo de dolor por cáncer sigue siendo la Escalera Analgésica de la OMS. 


\section{Distintos abordajes de clínicos quirúrgicos aplicando técnicas y procedimientos de acuerdo a criterios clínicos en oncología pediátrica}

Vol. 3, núm. 4., (2019)

Danixa Alexandra Rodríguez Chávez; María Antonia Dominguez Zambrano; Mirian Mariana Basurto Macías; Yadira Geovana Yanes Tigselena

Evaluación Neuropsicológica en Pacientes Oncológicos Pediátricos: Los

sobrevivientes de diversos cánceres producidos en la infancia tales como los tumores cerebrales y la leucemia, son susceptibles a múltiples tipos de deterioros cognitivos en los que se incluyen atención, memoria y velocidad para procesar información, todos estos como consecuencia del daño en los axones mielinizados en diferentes áreas del cerebro. De ahí, que el tratamiento de los tumores cerebrales se encuentra asociado con efectos adversos en el tejido sano del Sistema Nervioso Central lo que los hace muy propensos a desarrollar este tipo de deterioros. (Bestiet, 2017).

En los niños, el desarrollo del Sistema Nervioso Central no se ha completado y por lo tanto no han emergido todas las funciones cognitivas. Las alteraciones que se detectan no van a suponer una pérdida de la función, sino una disminución en las capacidades que debe desarrollar en comparación con su grupo de iguales. El cerebro del niño tiene la capacidad de recuperarse mejor de los daños focales, pero en daños generalizados el pronóstico es peor que en los adultos puesto que los niños suelen preservar funciones básicas pero la recuperación cognitiva no suele ser completa.

Las evaluaciones de seguimiento permiten ver el impacto del cáncer y sus tratamientos, ya que el daño en el SNC puedo no ser estático sino interrumpir los procesos de maduración por múltiples vías en detrimento de los hallazgos a largo plazo. Cuando se realiza una evaluación amplia, esta permite determinar la presencia no sólo de déficit específicos, sino también los sistemas funcionales que se encuentran intactos para así determinar la rehabilitación más oportuna para la posterior información a los padres de los pacientes. La exploración neuropsicológica es 


\section{Distintos abordajes de clínicos quirúrgicos aplicando técnicas y procedimientos de acuerdo a criterios clínicos en oncología pediátrica}

Vol. 3, núm. 4., (2019)

Danixa Alexandra Rodríguez Chávez; María Antonia Dominguez Zambrano; Mirian Mariana

Basurto Macías; Yadira Geovana Yanes Tigselena

fundamental para la evaluación del estado de las funciones cognitivas. Con esta, se puede detectar el daño producido en ellas por los tratamientos oncológicos, asimismo, la exploración resulta útil para detectar tumores o recidivas. (Bestiet, 2017).

Rehabilitación Neuropsicológica: Parte de una evaluación neuropsicológica que determina el posible deterioro, los déficits cognitivos específicos y los sistemas funcionales intactos. Es un área de investigación que no sólo abarca la recuperación de la función cognitiva deteriorada sino la compensación de la misma. El trabajo constante en rehabilitación neuropsicológica permite un feedback continuo que resulta clave en la promoción de calidad de vida de los niños que son afectados por tumores malignos. (Muller \& Mitthay, 2017).

Aspectos Psicológicos Asociados al Paciente Pediátrico con Cáncer: El diagnóstico de cáncer puede considerarse como un estresor potente que puede provocar reacciones emocionales, especialmente ansiedad y depresión e incluso trastornos emocionales del estado de ánimo. El objetivo fundamental debe ser conseguir que la información sobre el cáncer contribuya al proceso terapéutico y a la mejora de la calidad de vida de los pacientes. Entre los aspectos positivos que se pueden conseguir al informar el diagnóstico se encuentran, por un lado, respetar el derecho del paciente a ser informado, brindándole la posibilidad de conocer personalmente su realidad, diagnóstico, pronóstico y futuro. (Muller \& Mitthay, 2017).

Por otro lado, aquellos pacientes que han sido informados adecuadamente colaboran mejor en el tratamiento y también consiguen una mejor adaptación a su realidad, en especial a largo plazo, lo que ayuda a mejorar su calidad de vida y la de su entorno. En cuanto a los aspectos negativos, se evidencia que tanto el personal sanitario como los familiares del paciente oncológico, 


\section{Distintos abordajes de clínicos quirúrgicos aplicando técnicas y procedimientos de acuerdo a criterios clínicos en oncología pediátrica}

Vol. 3, núm. 4., (2019)

Danixa Alexandra Rodríguez Chávez; María Antonia Dominguez Zambrano; Mirian Mariana Basurto Macías; Yadira Geovana Yanes Tigselena

consideran que comunicarle el diagnóstico puede ser muy duro y es mejor protegerlo, su finalidad, es tratar de ahorrarle al paciente el estrés emocional que genera el diagnóstico. (Muller \& Mitthay, 2017).

El cáncer infantil tiene características propias que lo diferencian del cáncer en los adultos por lo que exigen tratamientos específicos. Los tipos de cáncer que más afectan a los niños son la leucemia, el linfoma, el tumor cerebral y los sarcomas de hueso y tejidos blandos, es por esto, que la enfermedad y el tratamiento proporcionado para ésta tiene fuertes implicaciones emocionales en los niños. El infante oncológico, experimenta los síntomas físicos de la enfermedad y del tratamiento como son las náuseas, vómitos, pérdida de peso o fatiga, entre otras, pero también se ve afectado por las repercusiones psicológicas. (Muller \& Mitthay, 2017).

Al conocer el diagnóstico los niños suelen preguntarse si van a morir, y en función de su edad, lograrán comprender en mayor o menor medida la enfermedad para así poder verbalizar sus sentimientos y sus miedos. Ante el diagnóstico de cáncer los niños más pequeños suelen manifestar principalmente su preocupación por el dolor y miedo a separarse de sus padres y de su entorno durante las hospitalizaciones. En los más grandes surgen sentimientos de soledad si la enfermedad no les permite participar de sus actividades diarias. (Muller \& Mitthay, 2017).

Los cambios corporales constituyen uno de los elementos que más causa preocupación por pérdida de autoestima y autoconfianza, que pueden llevar al niño a tener comportamientos regresivos, retraimiento de sus compañeros, miedo de ir al colegio, lo cual afecta las adaptaciones emocionales y el desarrollo académico. Esto generalmente sucede más en los adolescentes. El cáncer en la niñez produce estrés interna y externamente, porque altera la relación de los individuos 


\section{Distintos abordajes de clínicos quirúrgicos aplicando técnicas y procedimientos de acuerdo a criterios clínicos en oncología pediátrica}

Vol. 3, núm. 4., (2019)

Danixa Alexandra Rodríguez Chávez; María Antonia Dominguez Zambrano; Mirian Mariana

Basurto Macías; Yadira Geovana Yanes Tigselena

con su medio ambiente. Del mismo modo, los niños pueden sentirse alterados por el temor a las curas, a las batas blancas y a los instrumentos y procedimientos médicos, por la toma de determinados fármacos, por el sueño y por el dolor. En algunas ocasiones, el niño puede llegar a presentar ansiedad anticipatoria a los procedimientos médicos, que se manifiesta por náuseas, erupciones cutáneas, insomnio o llanto. (Muller \& Mitthay, 2017).

\section{Conclusiones.}

En la actualidad, el cáncer infantil se considera mayoritariamente una enfermedad crónica. Pese a esto, hoy en día se ha alcanzado una tasa actual de superviviendo de pacientes oncológicos de alrededor del 70\%, lo cual manifiesta que uno de cada 900 jóvenes que cumplen 20 años han sido sobrevivientes a algún tipo de cáncer en la infancia. El niño que sobrevive al cáncer puede verse expuesto según el diagnóstico y tratamiento realizado a diferentes riesgos que abarcan desde lo personal hasta lo social. Por ejemplo, son personas que tienen muy poco éxito académico, bajas tasas de empleo, bajas tasas de matrimonio y altos niveles de ansiedad en lo que se refiere a su fertilidad y a los riesgos que puedan correr con su salud.

Entre los tipos de cáncer que más riesgos presentan en sobrevivientes de cáncer en la edad adulta porque alteran su desempeño físico y restringen sus actividades de la vida diaria se encuentran los siguientes: cáncer cerebral, cáncer en los huesos, neuroblastoma, sarcoma en tejidos blandos y el linfoma de Hodgkin, siendo los sobrevivientes de cáncer en el cerebro y en los huesos los que presentan mayores restricciones. Los sobrevivientes de cáncer en los huesos y sarcoma en tejidos blandos son vulnerables a las limitaciones físicas y a algunas restricciones en su vida diaria 


\section{Distintos abordajes de clínicos quirúrgicos aplicando técnicas y procedimientos de acuerdo a criterios clínicos en oncología pediátrica}

Vol. 3, núm. 4., (2019)

Danixa Alexandra Rodríguez Chávez; María Antonia Dominguez Zambrano; Mirian Mariana Basurto Macías; Yadira Geovana Yanes Tigselena

debido a procedimientos quirúrgicos que como resultado pudieron crear daños o pérdidas de estructuras que son necesarias para la ejecución del movimiento.

Las limitaciones en el desempeño también son problemáticas en sobrevivientes del neuroblastoma primariamente porque estos son pacientes que tienen una multitud de efectos secundarios en el sistema musculoesquelético y en los sistemas neurológicos. Éstos a su vez, son los que tienen un riesgo de enfermedad bajo o intermedio debido a los tratamientos que se realizan en este tipo de pacientes tales como la cirugía radical, quimioterapia agresiva y radiación con rayos x. Entre las complicaciones musculoesqueléticas que presentan, se incluyen la escoliosis, la hipoplasia y fibrosis de tejidos blandos y huesos; entre los problemas neurológicos están las parestesias, las paresias y las paraplejías.

En los sobrevivientes del linfoma de Hodgkin por su parte, aunque se pueden presentar alteraciones que afectan su desempeño, no se dan en gran proporción como en los neuroblastomas, el cáncer cerebral y el cáncer en los huesos. Éstos en su gran mayoría presentan deterioros en el sistema cardiaco y pulmonar. Aunque la mayoría de los sobrevivientes aparentan tener una buena salud luego de todo el proceso de su enfermedad, solamente algunos de éstos llegan a tener grandes desajustes en lo que a su salud se refiere por lo que deben asistir a chequeos regulares que permitan identificar posibles problemas futuros en su salud.

En lo que respecta a la parte biológica se ha evidenciado que los efectos secundarios de la enfermedad y el tratamiento hacen que el niño sufra una serie de complicaciones que no sólo afectan su salud sino también su calidad de vida. Uno de los aspectos que ha recibido mayor atención por parte de los estudiosos es el manejo del dolor pues este es uno de los síntomas que 


\section{Distintos abordajes de clínicos quirúrgicos aplicando técnicas y procedimientos de acuerdo a criterios clínicos en oncología pediátrica}

Vol. 3, núm. 4., (2019)

Danixa Alexandra Rodríguez Chávez; María Antonia Dominguez Zambrano; Mirian Mariana

Basurto Macías; Yadira Geovana Yanes Tigselena

más afecta a los niños y sus padres. Es por esto que el personal médico debe estar alerta para conocer el tipo de dolor presentado por cada niño, recordando que en las diferentes fases del cáncer y el tratamiento se pueden experimentar diversos tipos de dolor que deben ser tratados con sumo cuidado para mejorar el estado del paciente pediátrico.

Otro de los problemas que se presenta en los niños con cáncer es la fatiga, siendo ésta un estado en la que el niño puede desarrollar sentimientos de impotencia, cansancio físico, mental y emocional que se atribuyen a los patrones de sueño experimentados por los niños, el ambiente hospitalario y los medicamentos empleados para el tratamiento. Por otra parte, las náuseas y vómitos que experimentan los niños enfermos de cáncer son resultado de las exposiciones a los tratamientos agresivos que tienen que afrontar para el tratamiento de su enfermedad lo que hace que no solamente sea un evento estresante, sino que también aparte del impacto emocional, causan un malestar físico pues las náuseas y vómitos traen como consecuencia efectos que pueden contribuir al desmejoramiento del estado de salud de los niños

\section{Bibliografía.}

Aguirre, P. (2016). Efectos de la enfermedad y su tratamiento en niños afectos de leucemia linfoblástica aguda. Revista Clínica y Salud de Puerto Rico, 6(3), 1-19.

Arias, F. (2010). Paradigmas de la Investigación Científica. España: Luces.

Bestiet, A. (2017). Efectos del tratamiento de Cáncer en pacientes Pediátricos. Psicooncología. Madrid. Aula de estudios sanitarios, 1-34.

Bradlyn, A. S., Beale, I. L., \& Kato, P. L. (2017). sychoeducational Interventions with Pediatric Cancer Patients. Part I. Patient Information and Knowledge. . Review: Journal of Child and Family Studies, 12(3), 257-277.

Garreto, G. (2016). El paciente Oncológico y su experiencia Clínica. Buenos Aires: Paidós. 


\section{Distintos abordajes de clínicos quirúrgicos aplicando técnicas y procedimientos de acuerdo a criterios clínicos en oncología pediátrica}

Vol. 3, núm. 4., (2019)

Danixa Alexandra Rodríguez Chávez; María Antonia Dominguez Zambrano; Mirian Mariana Basurto Macías; Yadira Geovana Yanes Tigselena

Marrown, J. (2017). Psicología Analítica en pacientes Oncológicos. Revista de Psicoteràpia Psiconalítica de México, 1-42.

Muller, S., \& Mitthay, K. (2017). Neuroblastoma: biology and staging. Curr Oncol Rep, 11(6), 431-8.

$$
\text { (C) (1) } Q(0)
$$

RECONOCIMIENTO-NOCOMERCIAL-COMPARTIRIGUAL

CC BY-NC-SA

ESTA LICENCIA PERMITE A OTROS ENTREMEZCLAR, AJUSTAR Y CONSTRUIR A PARTIR DE SU OBRA CON FINES NO

COMERCIALES, SIEMPRE Y CUANDO LE RECONOZCAN LA AUTORÍA Y SUS NUEVAS CREACIONES ESTÉN BAJO UNA LICENCIA CO N LOS MISMOS TÉRMINOS. 